\title{
Multiple Eruptive Sebaceous Hyperplasia Secondary to Cyclosporin in a Patient with Bone Marrow Transplantation
}

\author{
Begonia Cortés Gürkan Kaya \\ Division of Dermatology and Venereology, University Hospital of Geneva, \\ Geneva, Switzerland
}

\section{Keywords}

Sebaceous hyperplasia · Cyclosporin · Bone marrow transplant · Graft · Transplantation · Face

\begin{abstract}
Many cutaneous complications have been described in patients treated with cyclosporin. Alterations of the pilosebaceous unit such as hypertrichosis are particularly frequent. However, the occurrence of sebaceous hyperplasia is exceptional. These lesions seem to be specific to cyclosporin rather than secondary to immunosuppression. Here, we report an exceptional case of eruptive and disseminated sebaceous hyperplasia arising in a bone marrow transplant recipient only a few months after starting immunosuppressive treatment with cyclosporin.
\end{abstract}

\section{Introduction}

Sebaceous hyperplasia ( $\mathrm{SH}$ ) is a benign proliferation of the sebaceous glands, which in most cases is idiopathic and affects elderly patients. SH results from intrinsic, genetically determined (chronological) aging and extrinsic environmental photoaging. Moreover, this affection can arise in the setting of genetic diseases such as Muir-Torre syndrome or familial $\mathrm{SH}$. Some data reported in the literature suggest that cyclosporin could also be a causative factor [1]. We report an unusual case of eruptive multiple SH secondary to cyclosporin treatment in a bone marrow transplant recipient. 
Cortés and Kaya: Multiple Eruptive Sebaceous Hyperplasia Secondary to Cyclosporin in a Patient with Bone Marrow Transplantation

Fig. 1. Multiple asymptomatic skin-colored papules disseminated over the face and the neck (a-c).

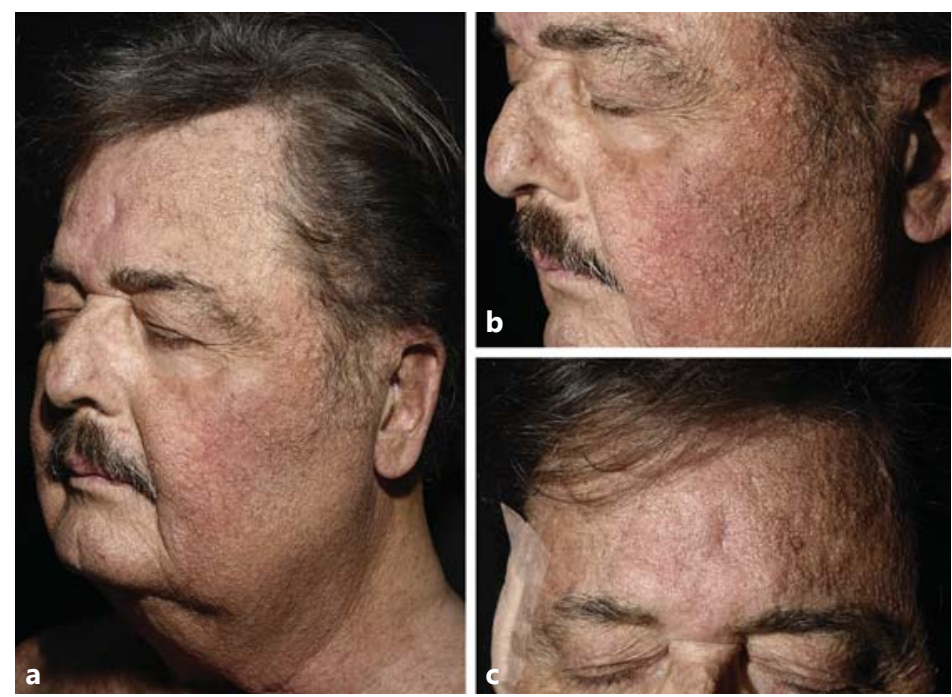

\section{Case Report}

A 69-year-old male with a diagnosis of acute myeloid leukemia received allogenic bone marrow transplantation in May 2014, after immunosuppressive treatment with high doses of chemotherapy and lymphoid and total body irradiation. Five months later, high doses of corticosteroids were introduced for an acute cutaneous graft-versus-host disease. After reducing the dosage of steroids, lichenoid lesions of the skin and oral mucosa consistent with a chronic graft-versus-host disease appeared. A treatment of cyclosporin $350 \mathrm{mg} /$ day $(4.4$ $\mathrm{mg} / \mathrm{kg}$ ) was initiated in December 2014, and the patient was then referred to our department to start extracorporeal photopheresis. Seven months later, he presented with multiple asymptomatic, skin-colored, 1- to 3-mm papules disseminated over the face and neck (Fig. 1). A skin biopsy of 1 of these lesions was performed. Histopathologic examination was consistent with $\mathrm{SH}$ with a cystic dilatation of the sebaceous duct (Fig. 2).

\section{Discussion}

Cyclosporin is an immunosuppressive drug that has been associated with several cutaneous adverse effects. These include tumoral lesions such as basal cell carcinoma and squamous cell carcinoma, which result from immunosuppression. Changes of the hair follicle specific to cyclosporin have also been observed. The most frequent manifestation is hypertrichosis, present in up to $60 \%$ of the patients. Other alterations of the pilosebaceous follicle include keratosis pilaris, acne, epidermal cysts, and folliculitis [2].

A few studies suggest that $\mathrm{SH}$ can also be a cutaneous manifestation specific to cyclosporin. SH was noted in 16 out of a total of 104 (16\%) heart transplant recipients. The condition occurred only in males and exclusively affected the face. The mean age of the patients with SH was 54 years [3]. Among kidney transplant recipients, 7 out of 67 (10\%) patients were reported to develop SH in another study. All of these patients were taking cyclosporin [2]. A few cases of disseminated SH secondary to cyclosporin therapy have been reported, among which there were 9 renal and 1 liver transplant recipients [4-9]. SH has been described in only 1 other bone marrow transplant recipient [10].

The development of benign and malignant cutaneous lesions in cyclosporin-treated transplant recipients is considered to be dose and duration dependent. In the majority of 
Cortés and Kaya: Multiple Eruptive Sebaceous Hyperplasia Secondary to Cyclosporin in a Patient with Bone Marrow Transplantation
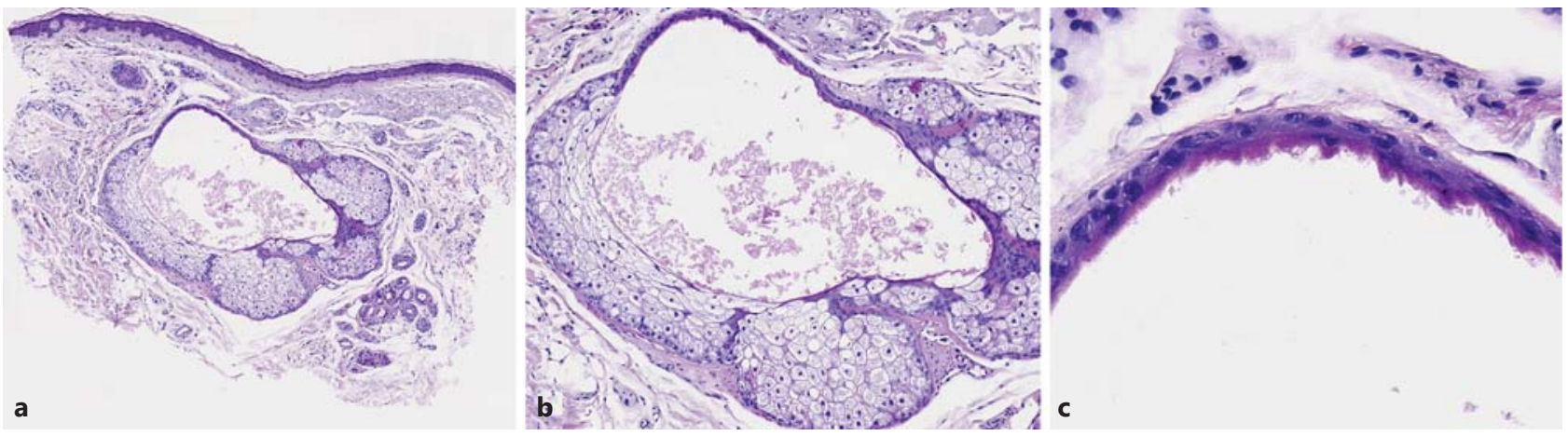

Fig. 2. Hyperplasia of the sebaceous gland with cystic dilatation of the sebaceous duct (a, b) showing characteristic crenulated keratinization (c).

cases, SH also occurs after many years of high-dose immunosuppressive therapy [6]. This contrasts with our patient who developed SH only a few months after starting cyclosporin treatment.

SH has not been observed in children, a fact that can be explained by the immaturity of the pilosebaceous unit in childhood [1]. Other immunosuppressive drugs have not been reported to induce SH in transplant recipients. This suggests that $\mathrm{SH}$ under cyclosporin is a direct and causal effect of this treatment on the sebaceous gland rather than a result of immunosuppression. Since cyclosporin is highly lipophilic, it has been suggested that the sebaceous gland might be the major cutaneous site of its elimination [4]. On the other hand, cyclosporine increases the activity of the 5 - $\alpha$ reductase enzyme, increasing sebaceous secretion. This could exert an occlusive follicular action and play a role in the pathogenesis of adnexal lesions [10].

The fact that only males are affected suggests a genetic basis. Treatment with immunosuppressive drugs may unmask latent Muir-Torre syndrome or promote tumor progression in genetically susceptible patients, leading to the development of SH.

Although the lesions are benign, they may have major cosmetic and psychological effects on the patients because they are located mainly in the facial region. Pulsed dye laser or carbon dioxide laser have been described to be a successful treatment of disseminated $\mathrm{SH}$, without recurrence after 6 months of follow-up despite continuation with cyclosporin treatment [8]. Isotretinoin inhibits sebaceous secretion and reduces the volume of the sebaceous gland. Some authors reported a good response to this molecule (10-20 mg/day) in renal transplant patients. After a few months of treatment, the lesions had almost completely disappeared. Nevertheless, patients had to be maintained on isotretinoin, as recurrence was common after the treatment had been stopped $[3,7,9]$. Other options include elctrodesiccation, shave excision, and photodynamic therapy [11].

\section{Conclusion}

In conclusion, $\mathrm{SH}$ is a cutaneous lesion that should be added to the possible rare but specific pilosebaceous changes occurring during cyclosporin treatment. Our case is exceptional because of the extension and the rapid development of the lesions. Moreover, these lesions are essentially described in renal transplant recipients. To our knowledge, this is only the second case of disseminated SH observed after bone marrow transplantation [10]. 


\section{Statement of Ethics}

The patient gave his informed consent for the publication of this work.

\section{Disclosure Statement}

The authors have no conflicts of interest to disclose.

\section{References}

1 Zouboulis CC, Boschnakow A: Chronological ageing and photoageing of the human sebaceous gland. Clin Exp Dermatol 2001;26:600-607.

2 Bencini PL, Montagnino G, Sala F, et al: Cutaneous lesions in 67 cyclosporin-treated renal transplant recipients. Dermatologica 1986;172:24-30.

-3 De Berker DAR, Taylor AE, Quinn AG, et al: Sebaceous hyperplasia in organ transplant recipients: Shared aspects of hyperplastic and dysplastic processes? J Am Acad Dermatol 1996;35:696-699.

4 Pang SM, Chau YP: Cyclosporin-induced sebaceous hyperplasia in renal transplant patients. Ann Acad Med Singapore 2005;34:391-393.

5 Boschnakow A, May T, Assaf C, et al: Ciclosporin A-induced sebaceous gland hyperplasia. Br. J. Dermatol 2003; 149:198-200.

6 Yayli S, Akyazi H, Bahadir S, et al: Coexistence of basal cell carcinomas and multiple sebaceous gland hyperplasias in a cyclosporine-treated renal transplant recipient. Am J Clin Dermatol 2010;11:59-62.

-7 Engel F, Ellero B, Woehl-Jaegle ML, Cribier B: Diffuse sebaceous hyperplasia of the face induced by cyclosporine. Ann Dermatol Venereol 2005;132:342-345.

-8 Truchuelo MT, Allende I, Almazán-Fernández FM, Boixeda P: Pulsed dye laser treatment for multiple sebaceous hyperplasia secondary to ciclosporin. Actas Dermosifiliogr 2011;102:470-471.

-9 McDonald SK, Goh MS, Chong AH: Successful treatment of cyclosporine-induced sebaceous hyperplasia with oral isotretinoin in two renal transplant recipients. Australas J Dermatol 2011;52:227-230.

10 Marini M, Saponaro A, Remorino L, et al: Eruptive lesions in a patient with bone marrow transplantation. Int J Dermatol 2001;40:133-135.

11 Simmons BJ, Griffith RD, Falto-Aizpurua LA, et al: Light and laser therapies for the treatment of sebaceous gland hyperplasia a review of the literature. JEADV 2015;29:2080-2087. 